\section{Prostate cancer in the Caribbean is taking down our men: access to care and changes in attitude are required}

\section{Dear Editor,}

The high rate of mortality from prostate cancer in the Caribbean poses a huge public health challenge for the area. As we know, non-communicable diseases (NCDs) are a growing challenge globally, as well as in the Caribbean (1). In fact, the Caribbean has the highest burden of NCDs in the Region of the Americas (2). Among these chronic diseases, several types of cancers have occupied the top causes of death.

In a recent study, researchers from the Caribbean Public Health Agency and the United States Centers for Disease Control and Prevention found that the rates of death from cervical, breast, prostate, and colon cancer are $2-9$ times higher in the Caribbean than in the United States (3). The study also reported that prostate cancer accounted for $18 \%-47 \%$ of cancer deaths. These figures are alarming considering that prostate cancer can be prevented through lifestyle changes and early detection and treatment.

Clearly, prostate cancer is a serious public health problem in the Caribbean, where its high incidence and mortality rates affect a predominantly Black population with an ancestral, genetic predisposition to the disease (4). But there are also many cultural and social norms that are proving to be obstacles to prevention and control among Caribbean men.

Caribbean men either do not adequately access health care or only access health care after signs and symptoms become severe (5). Many suffer in silence. Caribbean men generally have a love/hate relationship with health care. If they are not "sick," they do not access care; therefore, access to preventative services is decreased. If they are "sick," then they want care; that is, when signs and symptoms are obvious. This delayed access to care means prostate cancers are less likely to be detected in the early stages. Partially to blame is the "macho" culture that influences Caribbean men to suppress awareness of their bodies and not to show emotions or vulnerabilities. They often avoid seeking care until it becomes urgent. This leads to late detection.

Linked to this macho culture are a fear and avoidance of the digital rectal exam screening. Caribbean men continue to turn a blind eye towards getting tested. The 2007/8 Jamaica Health and Lifestyle Survey (6) showed that as many as $79 \%$ of men have never had a physical exam for prostate cancer, and only $13 \%$ reported having had one in the past 2 years.

While there are numerous non-modifiable risk factors associated with prostate cancer-genetics, age, race, and ethnicity-there are also several risk factors that are modifiable. They include smoking, diet, obesity, and sexually-transmitted infections, among others. Tobacco use is the largest, single contributor to cancer mortality. It causes a considerable number of deaths annually. The World Health Organization has projected that in 2020 , the overall prevalence of smoking will be $15 \%$ by people $\geq 15$ years of age in the Region of the Americas, with $19.2 \%$ among males compared to $10.8 \%$ among females (7).

To win the fight against prostate cancer, significant gaps must be filled. More research is needed on the importance of locallyrelevant, modifiable risk factors. With a greater understanding of these factors, preventive strategies can be instituted at both the population and individual levels.

In addition, there must be ways of getting men to access health care and to feel comfortable speaking with a health care provider about their ailments. Support systems must be put in place to encourage and support men in looking after their health.

Lastly, countries must implement effective, secondary, preventive strategies, such as mass screening. Other interventions should be urgently considered to bring prostate cancer under control and reduce not only the mortality, but the morbidity and accompanying burden. Our men must be cared for too!

Rosmond Adams

Health Information, Communicable Diseases, and Emergency Response Caribbean Public Health Agency (CARPHA) Port of Spain, Trinidad and Tobago \adamsros@carpha.org

Suggested citation Adams R. Prostate cancer in the Caribbean is taking down our men: access to care and changes in attitude are required [letter]. Rev Panam Salud Publica. 2018;42:e117. https://doi.org/10.26633/ RPSP.2018.117

\section{REFERENCES}

1. World Health Organization. Global status report on noncommunicable diseases. Geneva: WHO; 2010.

2. Healthy Caribbean. Non-communicable diseases (NCDs) in the Caribbean: the facts. Available from: www.archive.healthycaribbean.org/UNHLM-HCC/Caribbean-NCDs-Fact-sheet.pdf Accessed 25 August 2017.

3. Caribbean Public Health Agency. Breast cancer is two times higher in the Caribbean compared to the USA. Available from: http:// carpha.org/articles/ID/126/Breast-Cancer-is-Two-Times-Higher-inthe-Caribbean-Compared-to-the-USA Accessed 25 August 2017.

4. Zeigler-Johnson CM, Spangler E, Jalloh M, Gueye SM, Rennert H, Rebbeck TR. Genetic susceptibility to prostate cancer in men of African descent: implications for global disparities in incidence and outcomes. Can J Urol. 2008;15(1):3872-82.

5. Kaieteur News. Focusing on men's health [Editorial]. 30 January 2018. Available from: https://www.kaieteurnewsonline.com/2018/ 01/30/focusing-on-mens-health/ Accessed 22 June 2018.

6. Jamaica Health and Lifestyle Survey Research Group. Jamaica Health and Lifestyle Survey 2007-8. Technical Report. Mona, Jamaica: University of the West Indies; 2008. Available from: https:// www.paho.org/hq/dmdocuments/2012/Jamaica-Health-LifeStyleReport-2007.pdf Accessed 22 June 2018.

7. World Health Organization. WHO global report on trends in prevalence of tobacco smoking 2000-2025. $2^{\text {nd }}$ ed. Geneva: WHO; 2018. 